\title{
Autonomous Landing of Miniature Aerial Vehicles
}

D. Blake Barber

Stephen R. Griffiths

Timothy W. McLain

Brigham Young University - Provo, mclain@byu.edu

Randal W. Beard

Brigham Young University - Provo, beard@byu.edu

Follow this and additional works at: https://scholarsarchive.byu.edu/facpub

Part of the Mechanical Engineering Commons

\section{Original Publication Citation}

D. Blake Barber, Stephen R. Griffiths, Timothy W. McLain, and Randal W. Beard. "Autonomous Landing of Miniature Aerial Vehicles", Journal of Aerospace Computing, Information, and Communication, Vol. 4, No. 5 (2007), pp. 770-784. doi: 10.2514/1.26502

\section{BYU ScholarsArchive Citation}

Barber, D. Blake; Griffiths, Stephen R.; McLain, Timothy W.; and Beard, Randal W., "Autonomous Landing of Miniature Aerial Vehicles" (2007). Faculty Publications. 1498.

https://scholarsarchive.byu.edu/facpub/1498 accepted for inclusion in Faculty Publications by an authorized administrator of BYU ScholarsArchive. For more information, please contact ellen_amatangelo@byu.edu. 


\title{
Autonomous Landing of Miniature Aerial Vehicles
}

\author{
D. Blake Barber Stephen R. Griffiths Timothy W. McLain* Randal W. Beard \\ Brigham Young University Provo, UT 84602
}

This paper outlines an approach for automated landing of miniature aerial vehicles (MAVs). A landing algorithm defining the landing flight path as a function of height above ground, and the control strategies for following the path, are described. Two methods are presented for estimating height above ground, one based on barometric pressure measurements and the other utilizing optic-flow measurements. The development of an opticflow sensor and associated sampling strategies are described. Utilizing estimates of height above ground from barometric pressure and optic-flow measurements, repeated landings were performed with a $1.5 \mathrm{~m}$ wingspan MAV. With height above ground estimated from barometric pressure measurements alone, landing errors averaged $7.6 \mathrm{~m}$. When optic flow and barometric pressure measurements were combined to estimate height above ground, the average landing error was only $4.3 \mathrm{~m}$.

\section{Introduction}

The usefulness of miniature and micro air vehicles (MAVs) depends partly on the ability to hand launch and recover them in uncontrolled environments. Efforts to implement autonomous landing of MAVs in uncontrolled environments have been hindered by lack of small, lightweight sensors to determine height above ground (HAG). Because of the lack of a suitable sensor for determining HAG, MAVs have traditionally relied solely on barometric altimeters for HAG estimation. This approach works well for structured environments in which the altitude of the landing location relative to the altitude of the takeoff location is known, and the terrain is relatively smooth. However, in uncontrolled environments where this offset is not known, or where the terrain is not acceptably smooth, other methods must be utilized to effectively determine HAG.

One method for HAG estimation that has been recently proposed combines measurements of optic flow and ground speed. By correlating the optic flow of the ground observed from the MAV with the ground speed determined from GPS measurements, an estimate of the height of the MAV above the ground can be made. Estimates of HAG from optic flow can be combined with information from the barometric altimeter to determine HAG over a large altitude range. This estimate of HAG can then be utilized in landing control algorithms to safely land MAVs. This paper presents the experimental results of efforts to estimate HAG and land small fixed-wing MAVs in uncontrolled environments using an optic-flow sensor.

${ }^{*}$ Corresponding author, email: mclain@byu.edu 
Most of the work done regarding autonomous landing of small unmanned aerial vehicles (UAVs) has focused on the autonomous landing of unmanned rotorcraft. Most of these approaches involve using image processing techniques to estimate the pose of the UAV based on the motion of the aircraft relative to a structured target. $^{1,2}$ Researchers at the Army/NASA Rotorcraft Division have developed a method for landing an unmanned rotorcraft based on vision processing techniques, that does not require a structured reference image or structured landing environment. ${ }^{3,4}$

These techniques, though effective for rotorcraft, do not work well for fixed-wing MAVs. Development of robust landing algorithms for fixed-wing MAVs requires a sensor for estimating height above ground with acceptably small size and weight and high reliability. Recently, much of the research in this area has focused on utilizing vision processing techniques to estimate HAG. Chahl, et al. ${ }^{5}$ demonstrated that mimicking the landing behavior of bees, by maintaining constant optic flow during a landing maneuver, could be used to successfully control the descent of a MAV. Development of lightweight sensors for measurement of optic flow $^{6-8}$ has further bolstered the optic-flow-based approach. Barrows, et al. have demonstrated that these sensors can be successfully used to follow terrain in low flying MAVs. ${ }^{9}$

The use of optic-flow data for flight control is a subject of ongoing research. This is especially true for the autonomous landing of MAVs. Quigley, et al. presented an algorithm for the autonomous landing of MAVs utilizing barometric pressure sensing. ${ }^{10}$ Algorithms for augmenting this pressure-based method with data from optic flow are presented in this paper.

The landing algorithm utilized in this research is presented in section II. The longitudinal and lateraldirectional control laws used for automated landing are described in section III. Pressure-based HAG estimation and optic flow-based HAG estimation are discussed in section IV. Section V presents the flight test hardware used, while section VI presents automated landing results for MAVs using pressure-based and optic flow-based HAG estimation.

\section{Landing Algorithm Overview}

The landing algorithm developed and tested for this project requires two user-defined waypoints: the approach point and the landing point, as shown in Figure 1. The MAV orbits around the approach point as it descends to a specified altitude. At this end altitude, it breaks out of its orbit and follows a glide slope to the landing point. The approach point is fully defined by the following parameters:

1. Airspeed - The airspeed during the spiral-down portion of the landing.

2. Radius - The radius of the orbit during the spiral-down portion of the landing.

3. Start Altitude - The altitude to which the MAV climbs or descends while en route to the approach point. The true start altitude for the descent will be the altitude to which the MAV has successfully climbed or descended by the time it is within 1.5 orbit radii of the approach point.

4. End Altitude - The altitude at which the MAV breaks out of its orbit and begins its glide slope to the 
landing point.

5. Relative East Coordinate - The distance in the east direction from home to the approach point.

6. Relative North Coordinate - The distance in the north direction from home to the approach point.

7. Descent Rate - The rate at which the MAV descends while orbiting the approach point.

The landing point is fully defined by the following parameters:

1. Airspeed - The airspeed during the glide slope from approach point to landing point.

2. Flare Height - The height above ground at which the MAV cuts the throttle and attempts to hold zero roll while maintaining its glide slope by commanding pitch to control around desired altitude.

3. Relative East Coordinate - The distance in the east direction from home to the landing point.

4. Relative North Coordinate - The distance in the north direction from home to the landing point.

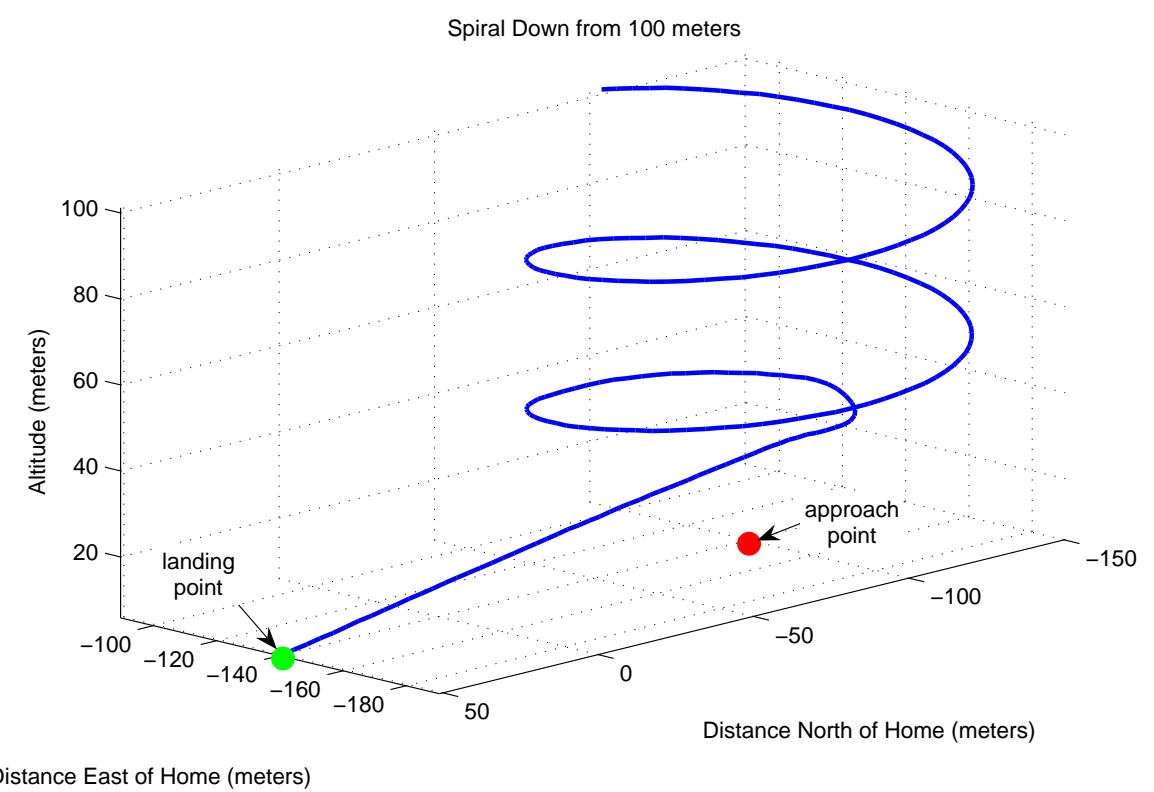

Figure 1. Landing spiral approach point and landing point.

\section{Control Approach}

\section{A. Altitude Control}

Altitude control throughout the landing routine is accomplished through activation of several PID loops. During the portion of the landing sequence that the MAV is en route from its last waypoint to the approach point, altitude is maintained according to the following criteria: 
1. If the actual altitude is within a preset distance (usually around $15 \mathrm{~m}$ ) of the desired altitude, then Pitch-from-Altitude and Throttle-from-Airspeed control laws are used. The Pitch-from-Altitude controller uses the altitude error to generate a pitch command for the pitch attitude control loop. The Throttle-from-Airspeed controller uses the airspeed error to generate a throttle command.

2. If the actual altitude is greater than the preset distance from the desired altitude and is less than the desired altitude, then the Pitch-from-Airspeed control law is used with full throttle. The Pitch-fromAirspeed controller uses the airspeed error to generate a pitch command for the pitch attitude control loop.

3. If the actual altitude is greater than the preset distance from the desired altitude and is greater than the desired altitude, then the Pitch-from-Airspeed control law is used with zero throttle.

For the portion of the landing sequence in which the MAV is spiraling down to its end altitude and then following its glide slope to the landing point, altitude is maintained using the Pitch-from-Altitude and Throttle-from-Airspeed loops. Throughout the entire landing sequence, until the flare at which desired roll is held at zero, course is controlled using the Roll-from-Course loop. Roll rate and pitch rate are also fed back to provide damping. Desired courses are commanded based on the generation of a course vector field around the desired orbit radius. A full description of this algorithm is provided in the following section.

The glide slope implemented in this landing routine was a simple linear glide slope calculated by decreasing desired altitude as a function of the distance from where the glide slope began. This formulation allows the desired altitude to be driven negative for distances from the initiation of the glide slope that are farther than the desired landing point. This makes the glide slope robust with respect to negative offsets between measured HAG and actual HAG. Expected overshoot or undershoot of the landing point can approximated as

$$
\text { expected over/undershoot } \approx \frac{1}{\tan \theta}\left(\tilde{h}+u_{h}\right),
$$

where $\theta$ is the glide slope, $\tilde{h}$ is the altitude control error (difference between desired and actual HAG), and $u_{h}$ is the measurement error. The relationship shown in Equation (1) indicates that larger glide slopes will tend to reduce the overall overshoot or undershoot error for the landing provided the MAV is capable of matching the commanded glide slope. However, larger glide slopes also cause increased impact velocities at touch down. Testing showed that glide slopes between 7 to 12 degrees provided a good balance between accuracy and impact at touch down for the MAVs flown.

\section{B. Orbit Routine}

\section{Course Vector Field Generation}

The ability to implement the landing routine described in Section II required the development and implementation of a control algorithm that would allow the MAV to fly accurate orbits, at minimal orbit radii, even in high-wind conditions. The orbit algorithm implemented generates a desired ground-track course $\left(\chi_{d}\right)$ 
based on the position of the MAV that directs the MAV onto the desired orbit as shown in Figure 2. Based on the position of the MAV, a field of desired course vectors can be generated using Equation (2).



Figure 2. Course field for a $50 \mathrm{~m}$ radius orbit.

$$
\chi_{d}= \begin{cases}\chi_{c}-\tan ^{-1}\left(\frac{r_{c}}{d}\right), & d \geq 2 r_{c} \\ \chi_{c}-\alpha-\beta \bar{r}^{\gamma}, & r_{c} \leq d \leq 2 r_{c} \\ \chi_{c}+\alpha+\pi+\beta \bar{r}^{\gamma}, & d<r_{c}\end{cases}
$$

In the above equations $d$ is the distance from the center of the orbit, $\chi_{d}$ is the desired course, $\chi_{c}$ is the course direction to the center of the orbit, $r_{c}$ is the desired orbit radius, $\alpha$ is the included angle between $\chi_{d}$ and $\chi_{c}$ for $d=2 r_{c}, \beta$ is the complimentary angle to $\alpha$, and $\gamma$ is a gain corresponding to the field strength of the orbit. Increasing $\gamma$ makes the field more strongly attracted to the desired orbit radius and decreasing $\gamma$ makes the field less strongly attracted as shown in Figure 3. $\bar{r}$ is a parameterization of the distance from the center of the orbit based on $r_{c}$. The formulation of $\bar{r}$ is such that $0 \leq \bar{r} \leq 1$, and $\bar{r}=1$ when $d=r_{c}$. The value of $\bar{r}$ is calculated according to Equation (3).

$$
\bar{r}= \begin{cases}1-\frac{d-r_{c}}{r_{c}}, & r_{c} \leq d \leq 2 r_{c} \\ \frac{d}{r_{c}}, & d<r_{c}\end{cases}
$$

The desired course from the course field described by Equation (2) is used to generate a roll command for the MAV according to

$$
\phi_{d}=k\left(\chi_{d}-\chi\right)
$$

where $\chi$ is the ground track course determined from GPS measurements. 


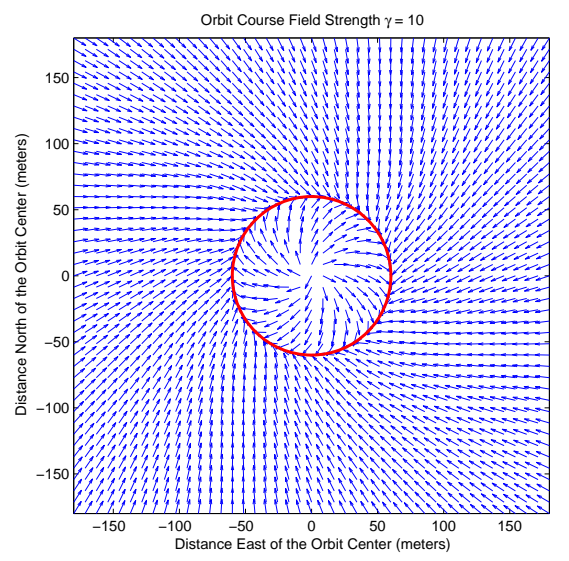

(a) High gain

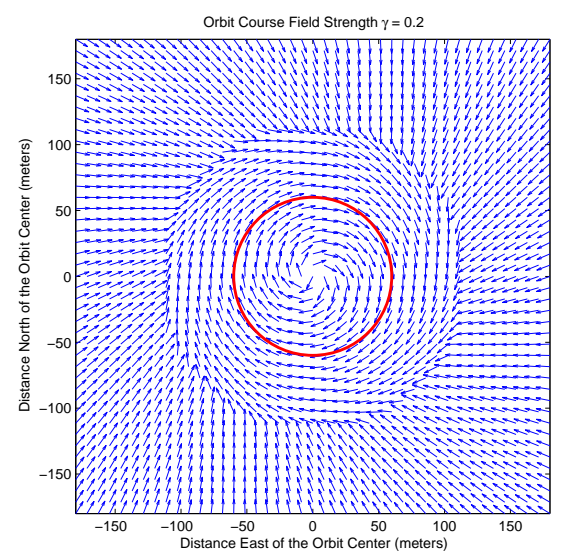

(b) Low gain

Figure 3. Course vector fields for a $50 \mathrm{~m}$ radius orbit.

\section{Steady-state Error Correction}

Because the course control system is type I and an orbit is a ramp in course, the course of the MAV tracks the desired course with a constant steady-state error. This steady-state error in course represents a lag between desired and actual course which translates to orbits that are larger than the commanded orbit. The magnitude of the steady-state error in orbit radius is a function of the desired radius of the orbit, the groundspeed of the MAV, and the course field gain $\gamma$. Two different approaches were used to reduce this error. The first approach involved adapting the radius command to remove the steady-state error. The second approach, which was more effective in high winds, involved calculating a nominal roll angle based on the desired course rate and the coordinated turn equation and adding that into the control as a feedforward command.

Adaptive Radius Commands Because the actual orbit radius of the MAV is always larger than the commanded radius, for every dynamically feasible orbit there exists some smaller radius command that will result in the desired orbit. The adaptive radius command progressively increases or decreases an offset between the desired orbit radius entered by the operator and the desired orbit radius passed to the orbit algorithm. This offset is continuously incremented or decremented until the MAV is orbiting at the radius requested by the operator. Orbit results from flight tests of the adaptive radius method are shown in Figure 4 .

Feedforward Roll Angle Based on Desired Course Rate The adaptive radius command approach works extremely well for situations where wind speed does not exceed 50 percent of airspeed. However, high winds cause the groundspeed of the MAV to vary significantly based on the course of the MAV relative to the wind direction. This variation in groundspeed causes a corresponding variation in the radius offset necessary to maintain the desired orbit. A better approach for high-wind conditions was developed that involved calculating a nominal feedforward roll angle command corresponding to the course rate required to fly the desired orbit. The feedforward roll command was used to augment the feedback command generated 


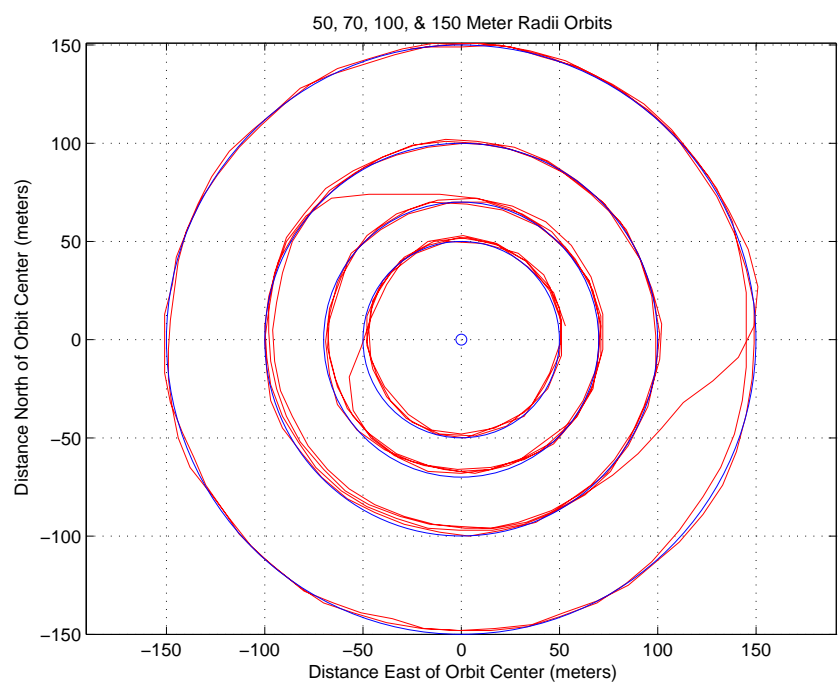

Figure 4. Flight test results for 50, 70, 100, and $150 \mathrm{~m}$ orbits flown in 2 to $3 \mathrm{~m} / \mathrm{s}$ wind.

from the course error. Calculating the feedforward roll command required taking the derivative of the course field with respect to time. Course rate is given by

$$
\dot{\chi}_{d}= \begin{cases}\frac{V r_{c}\left(r_{c}^{2}+2 d^{2}\right)}{d\left(r_{c}^{2}+d^{2}\right)^{\frac{3}{2}}}, & d \geq 2 r_{c} \\ \frac{V}{d} \sin \left(\alpha+\beta \bar{r}^{\gamma}\right)+\bar{r}^{(\gamma-1)}\left(\frac{\gamma \beta V \cos \left(\alpha+\beta \bar{r}^{\gamma}\right)}{r_{c}}\right), & r_{c} \leq d \leq 2 r_{c} \\ \frac{V}{d}\left(\sin \left(\alpha+\beta \bar{r}^{\gamma}\right)-\bar{r}^{\gamma} \gamma \beta \cos \left(\alpha+\beta \bar{r}^{\gamma}\right)\right), & d<r_{c} .\end{cases}
$$

Equation (2) and Equation (5) allow the calculation of both a desired course and a desired course rate for any location relative to the center of the orbit. The desired course rate can then be converted to a nominal roll angle using the standard coordinated turn equation

$$
\phi_{\text {nom }}=\frac{\dot{\chi}_{d} V}{g}
$$

and the control law described in Equation (4) can be modified to be

$$
\phi_{d}=\phi_{\mathrm{nom}}+k\left(\chi_{d}-\chi\right)
$$

The control law shown in Equation (7) is more effective than the adaptive radius command method at tracking orbits in high winds, but less steady at tracking orbits in low-wind conditions.

\section{Height Above Ground Estimation}

\section{A. Barometric Altimeter Based Landing}

The autopilot hardware used comes equipped with a barometric pressure sensor as part of its standard sensor suite. Before a given flight, the barometric pressure sensor is zeroed so that it references the barometric 
pressure at the takeoff location as the pressure corresponding to zero altitude. Pressure decreases with altitude according to

$$
P=P_{\text {ref }}-\int_{h_{\text {ref }}}^{h} \rho g h d h .
$$

If the density of air $(\rho)$ is assumed to be constant over the range of altitudes being measured, then altitude above the takeoff point can be determined by

$$
h=\frac{P_{\text {ref }}-P_{\text {actual }}}{\rho g}=\kappa\left(P_{\text {ref }}-P_{\text {actual }}\right),
$$

where the constant of proportionality $\kappa$ can be determined by calibration. Once the constant of proportionality has been determined, readings of the pressure sensor can be converted directly into relative altitudes above the takeoff point. This calibration allows the barometric pressure sensor to provide valid and accurate estimates of HAG where the terrain is flat. Where this is not true, barometric pressure can still provide a rough estimate of HAG until other sensors are able to collect more precise readings of the true HAG.

\section{B. Optic Flow Based Landing}

Optic flow sensors can be used to determine HAG by relating the flow of features across an imaging array to the speed the imaging array is moving past the surface it is imaging and its distance from that surface. The number of pixels that a given object moves in the imaging plane can be combined with data from the MAV's IMU and GPS to determine HAG according to

$$
h=\frac{\delta x}{2 \tan \left(\frac{\mu f}{2 p_{n}}-\frac{\dot{\theta} T_{s}}{2}\right)} \cos \theta \cos \phi,
$$

where $h$ represents HAG, $\delta x$ represents the displacement of the sensor parallel to the plane being imaged over the sample period $T_{s}, \mu$ represents the average number of pixels of displacement of features across the imaging plane over the same sample period, $f$ represents the field of view of the sensor, $p_{n}$ is the number of pixels in the imaging array in the direction of motion of the sensor, $\dot{\theta}$ is the average pitch rate of the sensor over the sample period, $\theta$ is the average pitch of the sensor over the sample period, and $\phi$ is the average roll of the sensor over the sample period.

SEnsor Testing of autonomous landing using optic flow to determine HAG was performed using Agilent's ADNS-2610 optic-flow sensor. The ADNS-2610 has a small form factor, measuring only $10 \mathrm{~mm}$ by $12.5 \mathrm{~mm}$ and runs at 1500 frames per second. It requires a light intensity of at least $80 \mathrm{~mW} / \mathrm{m}^{2}$ at a wavelength of $639 \mathrm{~nm}$ or $100 \mathrm{~mW} / \mathrm{m}^{2}$ at a wavelength of $875 \mathrm{~nm}$. The ADNS-2610 measures the flow of features across an 18 by 18 pixel CMOS imager. It outputs two values, $d x$ and $d y$, representing the total optic flow across the sensor's field of view in both the $x$ and $y$ directions. These values are stored in a buffer which can store accumulated optic flow up to 128 pixels. Once the buffer is full, no additional optic flow readings are accumulated until the buffer has been read. Reading the buffer clears it and allows it to begin reaccumulating optic flow measurements. The flow data in the camera $y$ direction corresponds to lateral motion of the MAV and can be ignored when estimating HAG. The flow data in the camera $x$ direction can be combined with data from the IMU and GPS to determine HAG according to Equation (10). 
Optics A critical consideration in outfitting a MAV with an optic-flow sensor is the setup of the optics. Narrow-angle lenses increase the distance at which the MAV is able to pick up appreciable optic flow. This increases the distance at which the sensor is able to accurately measure HAG for a given groundspeed. This comes at the expense of increasing the length of the sensor. Narrow-angle lenses can also decrease the low-end range of the sensor by causing overflow in the optic-flow sensor's $d x$ register or by causing the flow rate seen by the sensor to become too fast for the sensor to register. Wide-angle lenses allow for smaller longitudinal sensor size, but require that larger features be available in the environment. They also decrease the distance at which appreciable optic flow is recorded for a given groundspeed. A particular advantage of wider-angle lenses is that they are less susceptible to noise introduced by angular oscillations of the MAV in pitch and roll.

Another important consideration when designing the sensor optics is lens diameter. This is especially important with regard to the corresponding f-stop value of the lens. The f-stop is defined as the ratio of the lens focal length to its diameter and is a measure of the amount of light that the optics allow to pass through to the imaging array. Lenses with larger f-stops allow less light to pass through. Keeping in mind that the imaging array requires a light intensity of 80 to $100 \mathrm{~W} / \mathrm{m}^{2}$ in the correct spectral range, it is important to select a lens with a low f-stop in order to increase the sensor's operational range. Because the sensor automatically adjusts shutter speed to keep the light intensity at an ideal level, there is no disadvantage to selecting a lens with a lower f-stop other than increased size. Another important implication of the f-stop is that selecting a lens with an increased focal length (a narrower-angle lens) will not only increase the length of the sensor, but also increase the diameter of the sensor if the f-stop is to be maintained.

For the purposes of these tests, lenses were selected with fields of view of $6.5,2.5$, and 1.2 degrees when mounted on the sensor. These lenses had accompanying f-stops of 2.0, 2.0, and 2.5 respectively. Each of the configurations is shown in Figure 5. Comparable results were achieved using both the 2.5 degree and 1.2 degree field-of-view setups. However, the 1.2 degree field-of-view lens offered slightly greater range while performing better over feature-poor surfaces such as asphalt. One disadvantage of the 1.2 degree field-of-view setup up was its increased susceptibility to noise caused by pitch and roll oscillations.

Sample Rate Another critical consideration when equipping a MAV with an optic-flow sensor is the selection of sample rate. As expected, the sensitivity of the sensor changes with the HAG. We will show that the choice of sample rate can be used to mitigate this undesirable characteristic. If we consider the case of steady, level flight, height above ground is found from Equation (10) to be

$$
h=\frac{\delta x}{2 \tan \left(\frac{\mu f}{2 p_{n}}\right)} .
$$




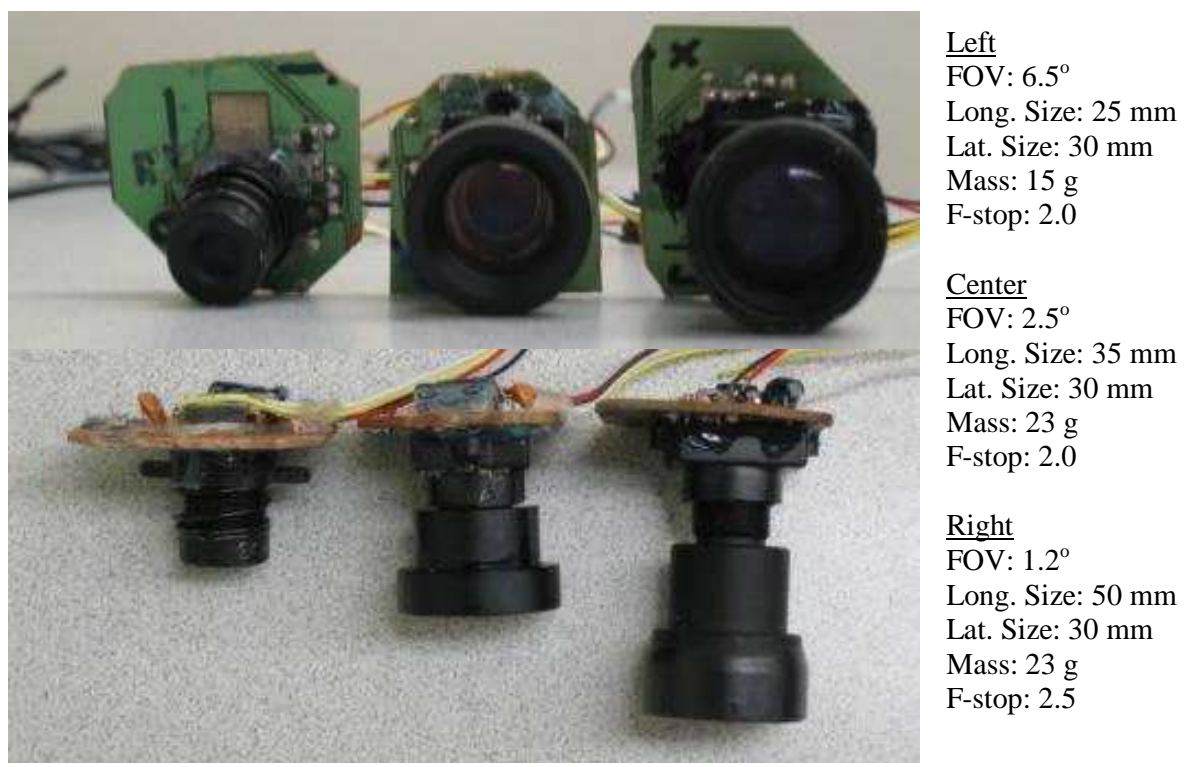

Figure 5. 1.2, 2.5, and 6.5 degree field of view optics configurations.

This expression shows how the ratio of sensor motion $(\delta x)$ to feature motion in the sensor image $(\mu)$ is used to determine HAG. We define the gain of the sensor (in this case, the inverse of its static sensitivity) to be

$$
\begin{aligned}
K & \triangleq \frac{d h}{d \mu} \\
& =-\frac{\delta x f}{4 p_{n}} \csc ^{2}\left(\frac{\mu f}{2 p_{n}}\right) \\
& =-\frac{V T_{s} f}{4 p_{n}} \frac{1}{\sin ^{2}\left[\tan ^{-1}\left(\frac{V T_{s}}{2 h}\right)\right]}
\end{aligned}
$$

where $V$ is groundspeed of the MAV. For a given sensor with a fixed field of view, the sensor's gain is a function of sample period, groundspeed, and HAG. Plots of sensor gain for a fixed field of view and varying sample rates and groundspeeds are shown in Figure 6. For the speeds, sample periods, and heights of interest, small angle approximations can be made and Equation (12) can be approximated accurately by

$$
K \approx-\frac{h^{2} f}{V T_{s} p_{n}} .
$$

Over the useful range of the optic-flow sensor, this approximation has an average error of 0.033 percent and a maximum error of 0.25 percent, which occurs when HAG is smallest.

Equation (13) shows how the gain of the sensor changes with HAG. The gain of the sensor also defines its resolution - the smallest HAG change that can be resolved by a single pixel change in the $d x$ output of the sensor. For high values of the gain, each sensor count corresponds to a relatively large distance measure. Correspondingly, the noise on the sensor (measured in sensor counts) is amplified by the gain in the HAG measurement. This makes large values of the sensor gain undesirable because they can significantly decrease the sensor's resolution and signal-to-noise ratio. It is possible to decrease the sensor gain by increasing the sample period as shown in Equation (13). While large sample periods may be desirable for large HAG 


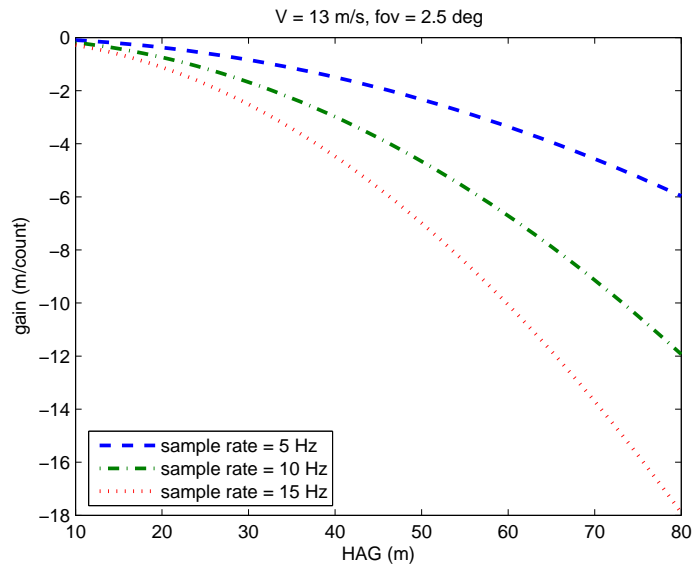

(a) Varied sample rates.

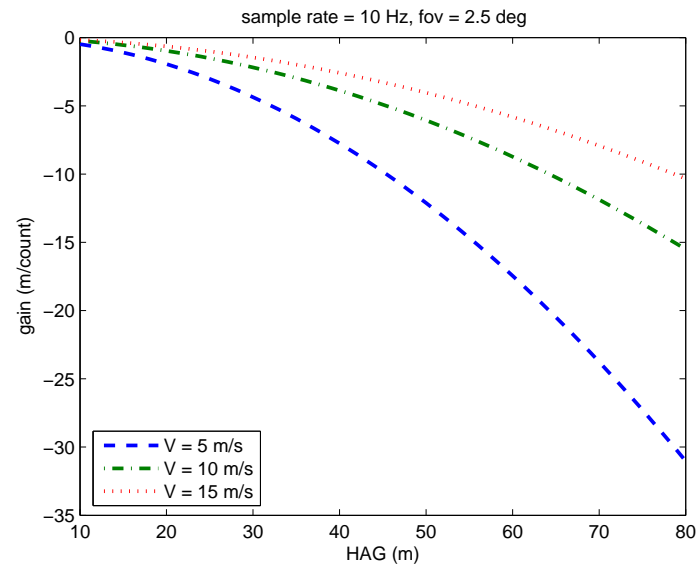

(b) Varied groundspeeds.

Figure 6. Sensor gain as function of HAG.

values, they can limit performance at low HAG values by slowing the sensor's response time and by causing overflows in the sensor's $d x$ register, thus limiting the minimum height that the sensor can measure. To solve these problems two approaches were developed using a dynamic rather than static sample rate. The first approach sets the sample rate to be proportional to the groundspeed, thus eliminating the influence of groundspeed on sensor gain. The second approach uses the current velocity and HAG estimates to maintain a constant value of sensor gain independent of groundspeed and HAG.

HAG-DEPEndent Gain In general, flight control systems for UAVs utilize a feedback loop to control around a commanded airspeed rather than groundspeed. Even when the feedback loop is closed around groundspeed, the commanded groundspeed may not be realizable due to wind conditions. In the presence of wind it is reasonable to assume that groundspeed may vary significantly depending on the heading of the MAV relative to the wind direction. As shown in Figure 6(b) and Equation (13), variations in groundspeed can alter the gain curve significantly. However, if the sample period is set according to

$$
T_{s}=\frac{\tau}{V},
$$

where $\tau$ represents the sample rate divisor, the sensor gain becomes a function of HAG only. This is useful because it eliminates the variations in gain due to groundspeed and provides reliable gains independent of wind conditions or commanded airspeeds.

Constant Gain When an estimate of the current HAG is available, the sample rate can be calculated so that the sensor gain can be maintained constant independent of HAG or groundspeed. This sample period is found by solving Equation (13) for $T_{s}$. The result is

$$
T_{s}=-\frac{h^{2} f}{V p_{n} K},
$$

where $K$ now represents a user-defined parameter that represents the desired gain of the sensor for all combinations of HAG and groundspeed. In practice, the value of $T_{s}$ is allowed vary to maintain a fixed 
sensor gain, but the range over which it may vary is capped at both the high and low ends. In flight tests, using a dynamic sample rate to fix sensor gain cut noise drastically for high values of HAG while significantly increasing the sample rate and thus improving the response time for low values of HAG.

Flight test results using both HAG-dependent gain and constant-gain approaches are shown in Figure 7. The spikes in the optic-flow measurement at sample 275 in the constant curve gain plot and 400 in the constant value gain plot correspond to flight over an asphalt road. In practice such spikes are ignored by discarding measurements for which the optic flow sensors report poor surface quality.

The advantage of the HAG-dependent gain approach is that a measurement of HAG is not required to set the sample period. The method is robust to variations in groundspeed, but the gain (and hence resolution) of the sensor will vary with changes in HAG. This is approach is most useful when the HAG is changing rapidly due to rough terrain. Note that although the gain and resolution of the sensor are changing, the average HAG over the sample period as determined by Equation (10) is valid. The advantage of the constant-gain approach is that the gain and resolution of the sensor can be held relatively constant over a wide range of HAGs and groundspeeds. The disadvantage of this approach is that it requires a good estimate of the HAG to set the sample period. This is not a problem when terrain variations are small over the sample period.

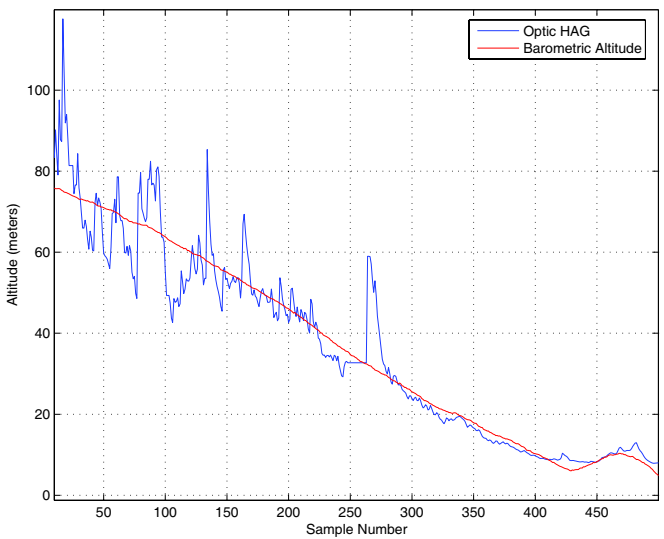

(a) HAG-dependent gain

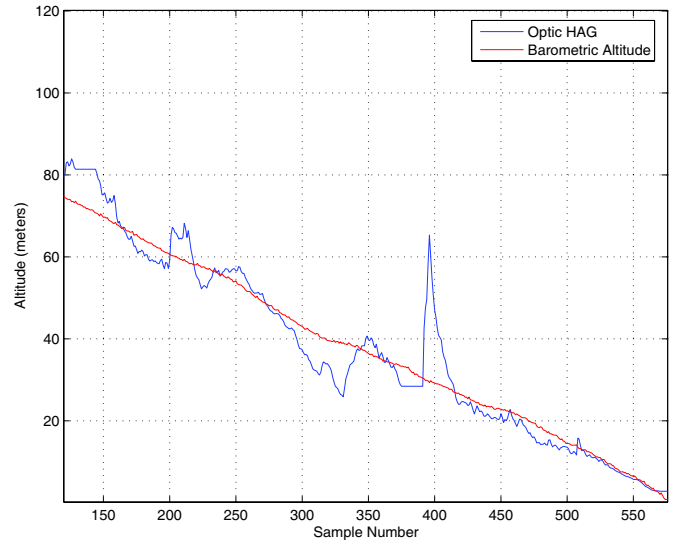

(b) Constant gain

Figure 7. Optic HAG results from glideslope descents.

The accuracy of the distance measurements calculated from optic flow was tested by comparison with a laser rangefinder. Testing was performed by mounting the optic-flow sensor and laser rangefinder perpendicular to the motion of a vehicle driving along a freeway at varying distances from the freeway sound barrier wall. Range values were recorded for both the optic-flow calculations and the laser rangefinder. The results of this test are shown in Figure 8. The results show close agreement and validate the readings obtained from the optic-flow calculations. Range data from the optic-flow sensor in flight tests showed reliable, repeatable readings for heights above ground less than $40 \mathrm{~m}$. For the purposes of landing based on HAG calculations from optic flow, values above $40 \mathrm{~m}$ were discarded as noise. 


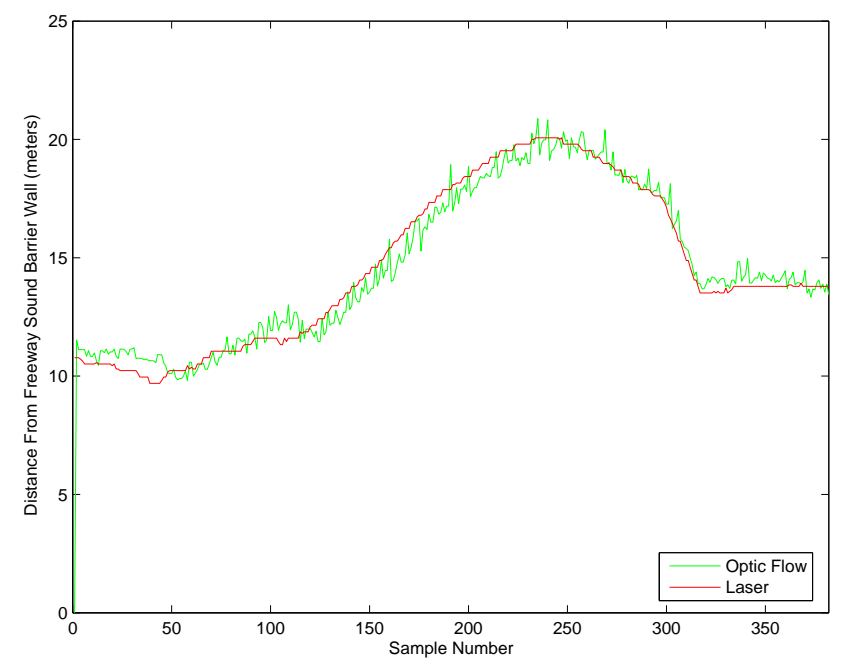

Figure 8. Optic flow range data compared to laser rangefinder data.

\section{Flight Test Hardware}

BYU has developed a reliable and robust platform for unmanned aerial vehicle testing. Figure 9 shows the key elements of the testbed. The first frame in Figure 9 shows a screen shot of the Virtual Cockpit ground station software. A laptop computer runs the Virtual Cockpit software that interfaces through a communication box to the MAVs. An RC transmitter is used as a stand-by fail-safe mechanism to facilitate safe operations.

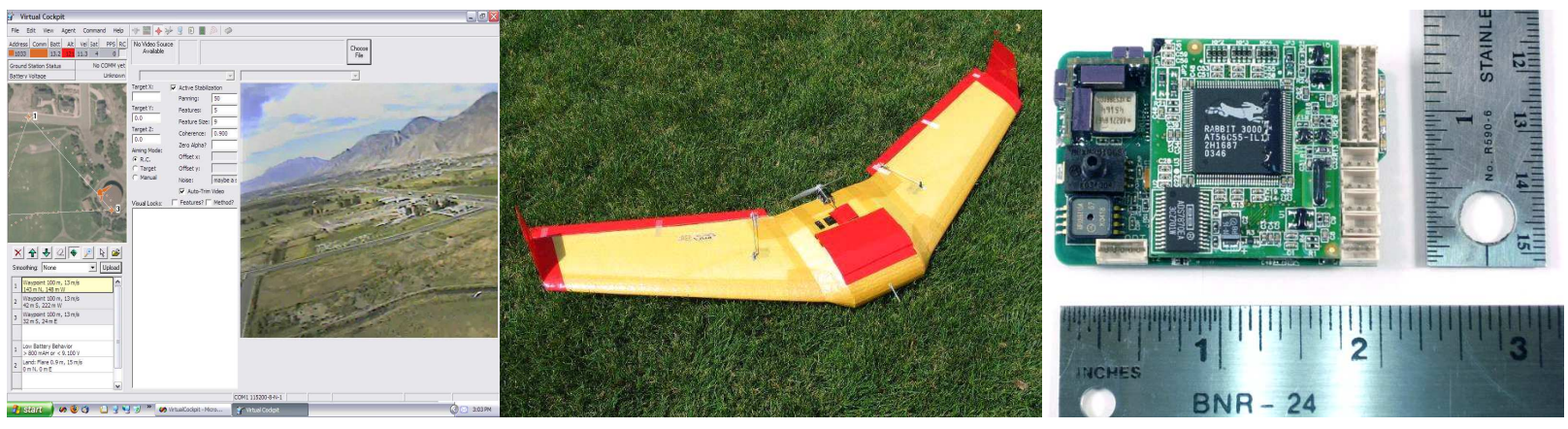

Figure 9. (a) Ground station components (b) Kevlar covered flying wing airframe (c) Kestrel autopilot.

The second frame in Figure 9 shows the airframe used for the flight tests reported in this paper. The airframe has a $1.5 \mathrm{~m}$ wingspan and was constructed with an EPP foam core covered with Kevlar. This design was selected for its durability, useable payload, ease of component installation, and flight characteristics. The airframe can carry a $0.8 \mathrm{~kg}$ payload and can remain in flight for over 90 minutes at a time. Embedded in the airframe are the Kestrel autopilot, batteries, a $1000 \mathrm{~mW}, 900 \mathrm{MHz}$ radio modem, a 12-channel GPS receiver, a video transmitter, a $400 \mathrm{~m}$ laser rangefinder, a small analog video camera, and three optic-flow sensors. 
The third frame shows BYU's Kestrel autopilot which is equipped with a Rabbit $340029 \mathrm{MHz}$ processor, rate gyros, accelerometers, and absolute and differential pressure sensors. The autopilot measures $3.8 \times$ $5.1 \times 1.9 \mathrm{~cm}$ and weighs 17 grams. The autopilot also serves as a data acquisition device and is able to $\log$ 175 kbytes of user-selectable telemetry at rates up to $60 \mathrm{~Hz}$.

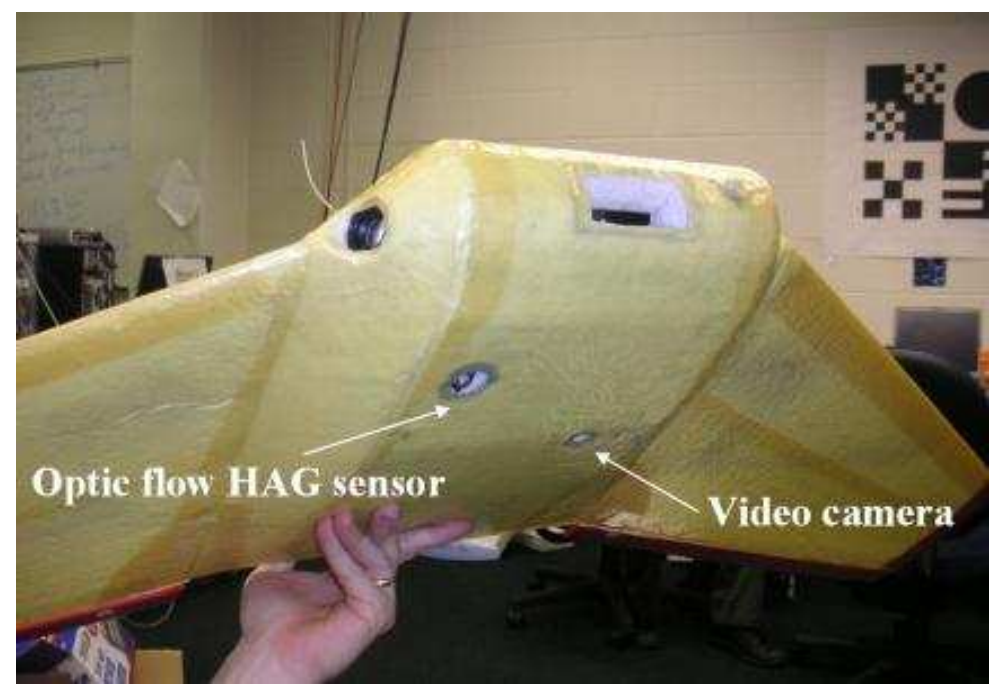

Figure 10. Optic HAG sensor and a downward pointed video camera on underside of airframe.

\section{Autonomous Landing Results and Discussion}

\section{A. Pressure Based HAG}

Using the calibration described by Equation (9), the MAGICC Lab MAVs consistently read within 1 to $1.5 \mathrm{~m}$ of the actual altitude relative to the home location. This allows relatively accurate landings where the altitude of the landing location can be safely assumed to be the same as the altitude of home location and works especially well when landing the MAV near its home location.

If the actual altitude of the MAV matched the desired altitude perfectly, then overshoot or undershoot would be solely a function of error in HAG estimation. For glide slopes of 7 to 12 degrees and a measurement error of 1 to $1.5 \mathrm{~m}$, a corresponding overshoot or undershoot error of around 4 to $12 \mathrm{~m}$ can be expected.

Results for 26 consecutive landing runs are shown in Figure 11. The first 22 autoland runs were each from the main approach point. These were followed by one run apiece from each of the four alternate approach points. An average overshoot or undershoot error of 4 to $12 \mathrm{~m}$ was estimated. Actual results from these 26 landing show an average over/undershoot error of $7.6 \mathrm{~m}$ with a standard deviation of $5.4 \mathrm{~m}$ and a median value of $6.0 \mathrm{~m}$. A video showing an autonomous landing using pressure-based altimetry is included with this paper. 


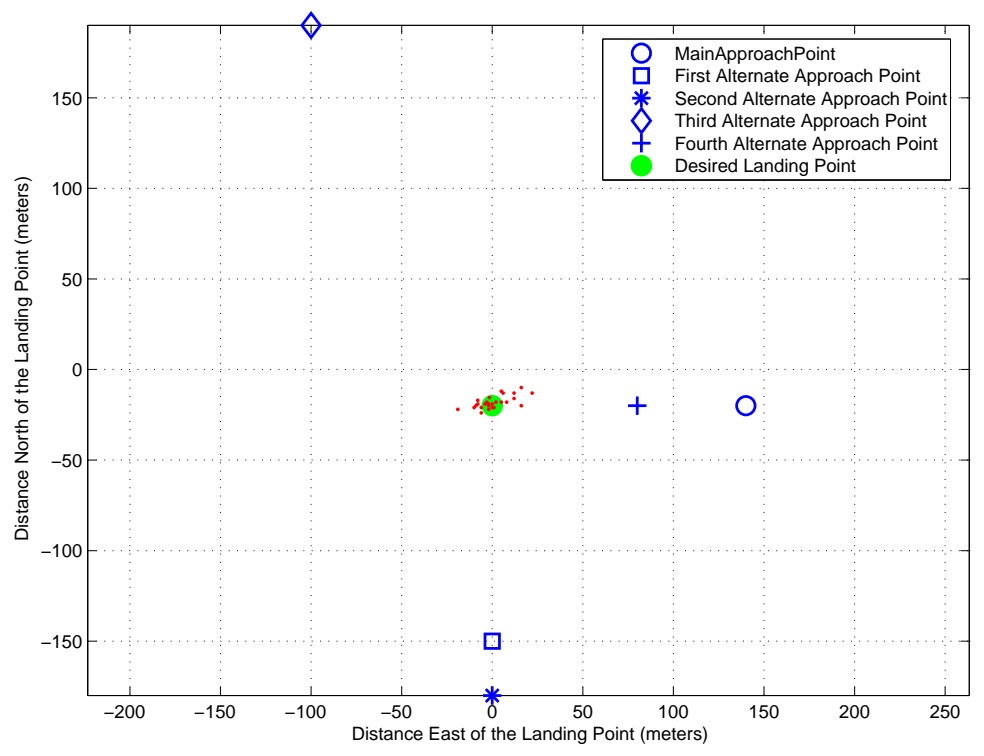

Figure 11. Pressure-based HAG autonomous landings.

\section{B. Optic Flow Based HAG}

Using the constant-gain sample rate calculation and the 2.5 degree field of view optics, HAG measurements based on optic flow were used to perform 27 consecutive autonomous landings from a single approach point. The results are shown in Figure 12. For each of these autonomous landings HAG was calculated according to

$$
\begin{aligned}
h & =h_{\mathrm{bar}}+h_{\mathrm{offset}} \\
h_{\mathrm{offset}} & =\nu\left(h_{\mathrm{bar}}-h_{\mathrm{optic}}\right)+(1-\nu) h_{\mathrm{offset}}
\end{aligned}
$$

where $\nu$ ranges between 0 and 1 . The value of $h$ was updated every time through the loop and the value of $h_{\text {offset }}$ was updated every $T_{s}$ seconds where $T_{s}$ varied with the current HAG estimate and groundspeed.

The readings from the barometric pressure sensor were allowed to drift over the 27 landings in order to simulate an unknown landing point altitude. The barometric altimeter drifted to $15 \mathrm{~m}$ of inaccuracy over the first 22 landings and was then rezeroed to introduce negative offsets between true HAG and barometric altitude. The value of $h_{\text {offset }}$ (corresponding to the drift in the barometric altimeter) for each of the landings is shown in the lower plot of Figure 13. The upper plot of Figure 13 shows the distance from the landing point for each of the 27 landings.

The average distance between the desired and actual touchdown points for autonomous landings using optic-flow data was $4.3 \mathrm{~m}$. This represents a 56 percent improvement over the average distance from the desired touchdown point using only the barometric altimeter. Landings based on HAG from optic flow also had a much narrower spread than those based on barometric altitude alone with a standard deviation of $2.2 \mathrm{~m}$ for optic flow based landings compared to $5.4 \mathrm{~m}$ for barometric. 


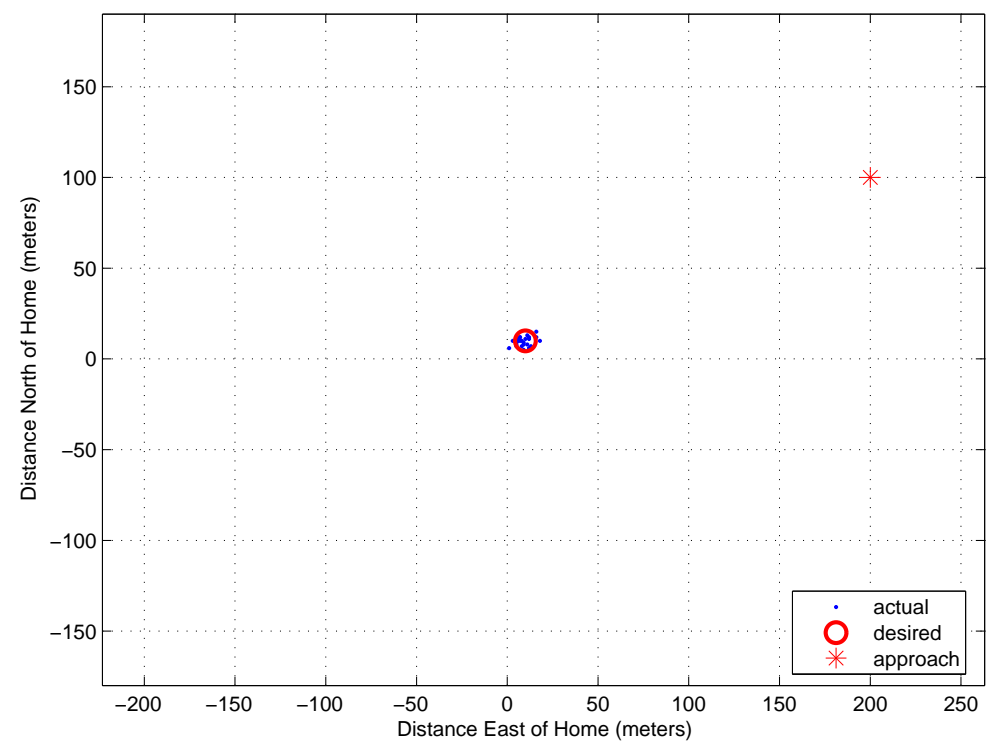

Figure 12. Optic flow-based HAG autonomous landings.
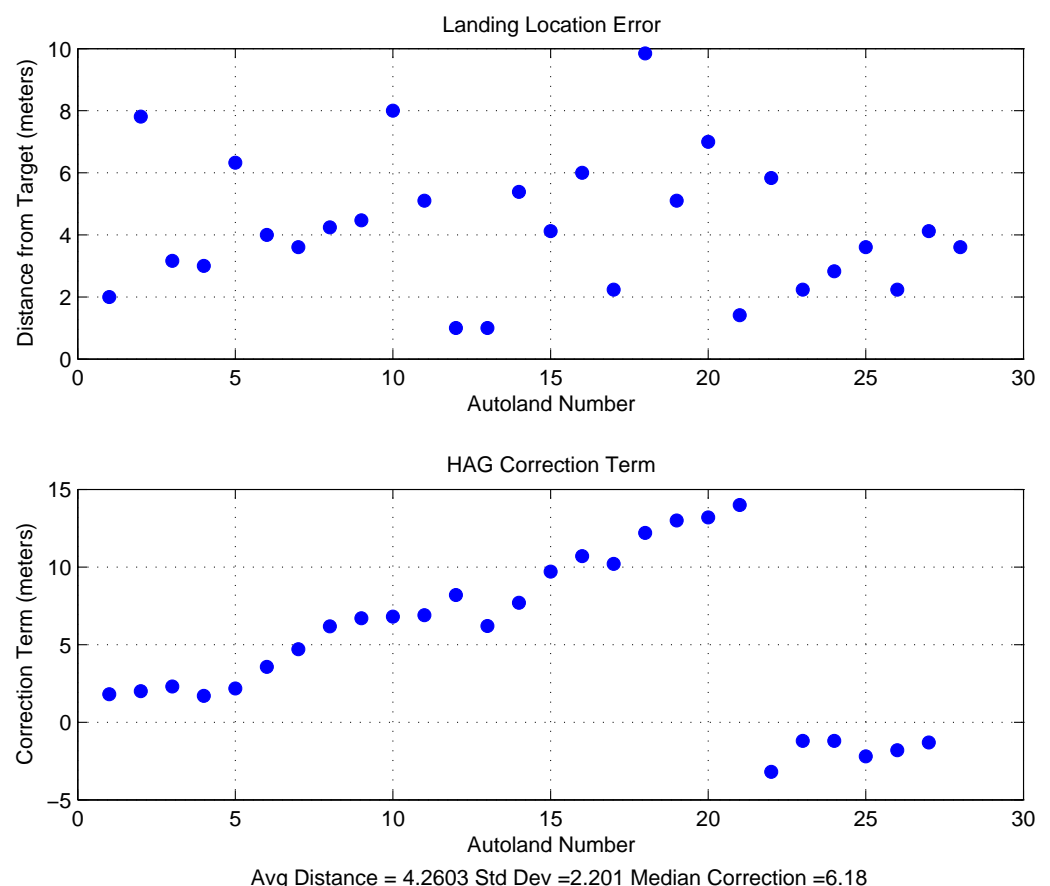

Figure 13. Landing error and HAG correction.

16 of 18

American Institute of Aeronautics and Astronautics 
Because the spiral down portion of the landing routine is based on barometric altitude until HAG readings fall under the $40 \mathrm{~m}$ threshold, it is important to note that the distance from the desired touchdown point does not increase with increasing error in barometric altitude. This demonstrates the ability of the optic flow sensor to land the MAV accurately even when the altitude of the landing point relative to the takeoff point is not known.

\section{Conclusions}

A simple algorithm for performing autonomous MAV landings has been presented. This landing algorithm utilizes a lateral-directional control method based on course vector fields. This algorithm has been tested both in simulation and in hardware and has proven effective, repeatable, and robust to wind. The algorithm has been used to perform hundreds of autonomous landings in a wide variety of wind conditions.

This paper demonstrates that the precision of autonomous landing can be enhanced by direct measurement of height above ground. In environments where the terrain is flat and the altitude of the landing point relative to the takeoff point is known, a well-calibrated barometric altimeter is sufficient to accurately determine HAG. In environments where this is not the case, fusion of data from the barometric altimeter and the optic-flow sensor provides a feasible and robust method for estimating HAG. When utilized by the landing algorithms presented in this paper, automated landings to within several meters can be performed repeatably.

\section{Acknowledgments}

This work was funded by AFRL/MNK award number F08630-03-1-0017.

\section{References}

\footnotetext{
${ }^{1}$ Shakernia, O., Vidal, R., Sharp, C. S., Ma, Y., and Sastry, S., "Multiple View Motion Estimation and Control for Landing an Unmanned Aerial Vehicle," Proceedings of the 2002 IEEE International Conference on Robotics ES Automation, Washington DC, May 2002, pp. 2793-2798.

${ }^{2}$ Sharp, C. S., Shakernia, O., and Sastry, S. S., "A Vision System for Landing an Unmanned Air Vehicle," Proceedings of the 2001 IEEE International Conference on Robotics \& Automation, Seoul, May 2001, pp. 1720-1727.

${ }^{3}$ Hintze, J., Christian, D., Theodore, C., Tischler, M., and McLain, T., "Automated Landing of a Rotorcraft UAV in a Noncooperative Environment," Proceedings of the 60th Annual Forum of the American Helicopter Society, Baltimore, Maryland, June 2004.

${ }^{4}$ Theodore, C., Sheldon, S., Rowley, D., McLain, T., Dai, W., and Takahashi, M., "Full Mission Simulation of a Rotorcraft Unmanned Aerial Vehicle for Landing in a Non-Cooperative Environment," Proceedings of the 61st Annual Forum of the American Helicopter Society, Grapevine, Texas, June 2005.

${ }^{5}$ Chahl, J., Srinivasan, M., and Zhang, S., "Landing Strategies in Honeybees and Applications to Uninhabited Airborne Vehicles," The International Journal of Robotics Research, Vol. 23, No. 2, 2004, pp. 101-110.

${ }^{6}$ Barrows, G. and Neely, C., "Mixed-mode VLSI optic flow sensors for in-flight control of a micro air vehicle," Proceedings SPIE, San Diego, August 2000, pp. 52-63.
}

17 of 18 
${ }^{7}$ Ruffier, F. and Franceschini, N., "Visually Guided Micro-Aerial Vehicle: automatic take off, terrain following, landing and wind reaction," Proceedings of the 2004 IEEE International Conference on Robotics \& Automation, New Orleans, 2004, pp. 2339-2346.

${ }^{8}$ Zufferey, J.-C. and Floreano, D., "Toward 30-gram autonomous indoor aircraft: Vision-based obstacle avoidance and altitude control," Proceedings of the 2005 IEEE International Conference on Robotics 83 Automation, Barcelona, April 2005, pp. 2594-2599.

${ }^{9}$ Barrows, G. L., Chahl, J. S., and Srinivasan, M. V., "Biomimetic Visual Sensing and Flight Control," The Aeronautical Journal, London: The Royal Aeronautical Society, Vol. 107, No. 1069, 2003, pp. 159-168.

${ }^{10}$ Quigley, M., Barber, D. B., Griffiths, S., and Goodrich, M. A., "Towards Real-World Searching with Fixed-Wing MiniUAVs," Proceedings of the 2005 IEEE/RSJ International Conference on Intelligent Robots and Systems, Edmonton, Alberta, August 2005, pp. 3028-3033.

18 of 18 